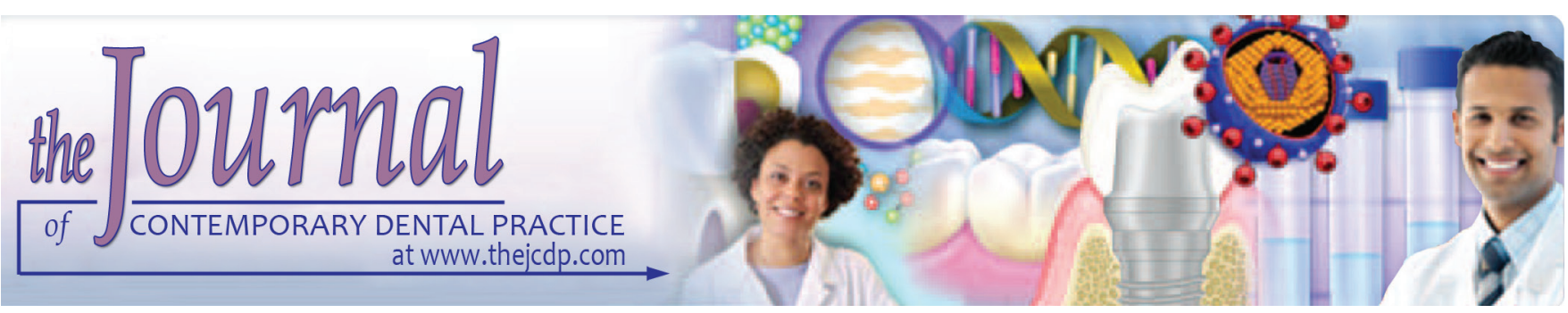

\title{
A Cross-sectional Study to assess the Perception of Psychosocial Elements among Pediatric Patients visiting Dental Clinics
}

\author{
${ }^{1}$ Ashish Bhalla, ${ }^{2}$ UB Rajasekaran, ${ }^{3}$ Madhavi Singh, ${ }^{4}$ Manish Goutam, ${ }^{5}$ Nikhil Grover, ${ }^{6}$ Ashok Galav
}

\begin{abstract}
Introduction: Children with high dental fear and perception usually encounter high prevalence of dental caries. Data showed that individuals with dental fear will probably defer dental appointments which may result in the formation of a setup of an endless loop of dental fear. Present study was aimed to assess different psychosocial parameters among pediatric patients visiting dental clinics.
\end{abstract}

Materials and methods: In the present cross-sectional study, the data of 200 schoolchildren were collected and analyzed by means of a questionnaire. Evaluation of the oral health status was done by experienced pedodontists. All the answers were recorded and analyzed by Statistical Package for the Social Sciences (SPSS) software.

Results: Nearly 120 were regular attendees while remaining 80 were found to be irregular attendees. Significant difference was observed while comparing the decayed, missing, and filled teeth (DMFT) index, decayed teeth, dental fear, cognitive vulnerability, and dental prevention belief between the regular and irregular group. Significant correlation was observed between higher number of decayed teeth and increasing level of dental fear.

${ }^{1}$ Department of Public Health Dentistry, Rama Dental College Hospital \& Research Center, Kanpur, Uttar Pradesh, India

${ }^{2}$ Department of Preventive Dentistry and Orthodontics, Faculty of Dentistry, Sebha University, Libya

${ }^{3}$ Department of Periodontics, Dentica Oral Care and Implant Centre, Ranchi, Jharkhand, India

${ }^{4}$ Department of Prosthodontics, Dentica Oral Care and Implant Centre, Ranchi, Jharkhand, India

${ }^{5}$ Department of Pedodontics and Preventive Dentistry, School of Dental Sciences, Sharda University, Greater Noida, Uttar Pradesh, India

${ }^{6}$ Department of Oral Diagnosis, Medicine and Radiology Tatyasaheb Kore Dental College \& Research Centre, Kolhapur Maharashtra, India

Corresponding Author: Ashish Bhalla, Department of Public Health Dentistry, Rama Dental College Hospital \& Research Center, Kanpur, Uttar Pradesh, India, Phone: +919453534382 e-mail: asish070185@gmail.com
Conclusion: Various psychosocial parameters, by affecting the dental visits, are associated with outcome of oral health measures.

Clinical significance: Special attention should be paid to the pediatric patients with such kind of behavior so that their psychosocial attitude could be modified in favor of dental treatment.

Keywords: Caries, Pediatric, Psychosocial.

How to cite this article: Bhalla A, Rajasekaran UB, Singh M, Goutam M, Grover N, Galav A. A Cross-sectional Study to assess the Perception of Psychosocial Elements among Pediatric Patients visiting Dental Clinics. J Contemp Dent Pract 2017;18(11):1021-1024

Source of support: Nil

Conflict of interest: None

\section{INTRODUCTION}

Individuals with high dental dread and dental fears regularly encounter a scope of aversive mental, enthusiastic, and social issues. Albeit, dental dread is a diagnosable mental state with related mental side effects, which additionally has imperative and testing physical well-being suggestions. ${ }^{1-3}$ Individuals with dental dread frequently have poorer oral hygiene status in comparison with individuals with no dental dread, and at times, the long haul postponement of dental treatment may prompt the advancement of oral torment and the requirement for intrusive and conceivably difficult dental treatment. ${ }^{4}$

Investigations has reliably demonstrated that individuals with dental fear will probably defer dental arrangements and this may result in the formation of a setup of an endless loop of dental dread. Delayed dental visits permit the movement of oral malady which may prompt the prerequisite for crisis treatment which then serves to intensify or keep up the individual's dental fear. ${ }^{5}$ Dental dread has a high pervasiveness in numerous Western nations, yet it is the genuine oral well-being results of dental dread that stand separated from numerous other 
Table 1:Correlation of various parameters between regular and irregular attendees

\begin{tabular}{lllll}
\hline Parameter & Total $(n=200)$ & $\begin{array}{l}\text { Regular attendees } \\
(n=120)\end{array}$ & $\begin{array}{l}\text { Irregular attendees } \\
(n=80)\end{array}$ & $p$-value \\
\hline DMFT index & 2.12 & 1.92 & 2.26 & $0.02^{*}$ \\
Decayed teeth & 1.42 & 1.01 & 2.01 & $0.03^{*}$ \\
Dental fear (between 1 and 5) & 1.55 & 1.60 & 1.50 & $0.01^{*}$ \\
Cognitive vulnerability perception (between 1 and 4) & 1.75 & 1.92 & 1.61 & $0.02^{*}$ \\
Dental prevention belief (between 1 and 5) & 4.25 & 4.50 & 4.02 & $0.03^{*}$ \\
\hline
\end{tabular}

*Significant

particular feelings of dread and which make it such an essential region of study. ${ }^{6,7}$

Hence, we undertook the present study to assess different psychosocial parameters among pediatric patients visiting dental clinics.

\section{MATERIALS AND METHODS}

The present study was conducted in the Department of Public Health Dentistry and Department of Pediatric and Preventive Dentistry of the dental institution and included assessment of 200 pediatric patients from June 2014 to July 2016. All the individuals recruited in the present study were obtained from two different schools of the region. Of 200 pediatric individuals, 120 were males and 80 were females. Mean age of the individuals in the present study was 13.5 years. Ethical approval was taken from the institutional ethical committee, and written consent was obtained from the parents/guardians of all the individuals after explaining in detail the entire research protocol. Detailed informative educational sessions were given to all the children as a part of group discussion, which was followed by oral hygiene checkup of all the individual participants. No treatment protocol was started or done during the first visit. A questionnaire was prepared and given to all the pediatric individuals before starting the information session. The questionnaire was specially prepared for recording the oral health beliefs, dental-related various kinds of fear, and various measures of oral habits of the individuals. The questionnaire forms were filled by all the individuals in the presence of the research team and pedodontists. Assessment of the incidence of dental visits was done as a single item (what is your frequency of dental visit?) which had five options:

1. Never visited a dentist

2. Visited dentist only at the time of dental pain

3. Half yearly

4. Once yearly

5. Once in every 3 years

Following scoring was done for the participant's response to dental attendance:

- Regular attendance: Scored as 1 (half yearly or every year visit to dentist)

- Irregular attendance: Scored as 0 (visited dentist only at the time of dental pain and once in every 3 years)
For the assessment of dental fear and anxiety, index of dental fear and anxiety was used, which consisted of eight different items. ${ }^{8}$ These measured the psychological, cognitive, emotional, and behavioral components of dental anxiety. ${ }^{8}$ Oral well-being status of the pediatric patients was assessed by pedodontists, blinded to the review's motivation and autonomous from the examination group, utilizing a front-surface mouth reflect, test, cloth, and packed air, under fitting simulated light conditions. Specific instructions were given to the dentists for reporting the World Health Organization (WHO) index for missing and filled permanent teeth (DMFT). ${ }^{8}$ All the data were collected and summarized. All the results were analyzed by SPSS software version 18.0. Chi-square test, Student's t-test, Pearson's correlation, and descriptive analysis were used for the assessment of level of significance; $p<0.05$ was taken as significant.

\section{RESULTS}

Correlation of various parameters between regular and irregular attendees is shown in Table 1. Out of total 200 individuals, 120 were regular attendees, while remaining 80 were irregular attendees. Decayed, missing, and filled teeth index in the regular and irregular attendees group was found to be 1.92 and 2.26 respectively. As far as dental fear is concerned, the mean values observed in the regular and irregular group were found to be 1.60 and 1.50 respectively. Significant difference was observed while comparing the DMFT index, decayed teeth, dental fear, cognitive vulnerability, and dental prevention belief between the regular and irregular group $(p<0.05)$. Table 2 shows the Pearson's correlation between different

Table 2:Pearson's correlation between different parameters

\begin{tabular}{llllll}
\hline Parameters & One & Two & Three & Four & Five \\
\hline Age & - & - & - & - & - \\
DMFT & $0.39^{*}$ & - & - & - & - \\
Decayed teeth & $0.35^{*}$ & $0.90^{*}$ & - & - & - \\
Dental fear & $0.21^{*}$ & $0.17^{*}$ & $0.19^{*}$ & - & - \\
Cognitive vulnerability & $0.17^{*}$ & 0.02 & $0.10^{*}$ & $0.51^{*}$ & - \\
perception & & & & & \\
Dental prevention & $-0.15^{*}$ & -0.04 & -0.13 & $-0.25^{*}$ & -0.24 \\
belief & & & & & \\
\hline
\end{tabular}

*Significant $(p<0.05)$ 
parameters. Significant correlation was observed between higher number of decayed teeth and increasing level of dental fear.

\section{DISCUSSION}

Dread of and nervousness toward going to dental specialists (i.e., dental dread and uneasiness) are significant issues for a sizeable extent of youngsters and teenagers. ${ }^{9}$ The predominance of dental dread and uneasiness in kids and young people ranges from 5 to $20 \%$ in different nations, with a few cases being thought to be dental fear. Kids and teenagers with dental dread and nervousness are frequently uncooperative amid dental visits, by rendering treatment troublesome or inconceivable. ${ }^{10,11}$ Such conduct bargains the treatment result and makes a reason for dissension between dental experts and patients or their relatives. Dreadful youngsters and teenagers may attempt to stay away from or defer treatment, bringing about crumbling their oral well-being. Past ill dental experiences, dental dread and uneasiness may likewise bring about rest issue, influence one's everyday life, and negatively affect one's psychosocial working. Dental dread and tension obtained in youth may persevere to adulthood and is a noteworthy indicator for shirking of dental visits in adulthood. ${ }^{12,13}$

Hence, we undertook the present study to assess different psychosocial parameters among pediatric patients visiting dental clinics.

In the present study, we observed that significant difference existed between the regular and the irregular group while comparing DMFT index, dental fear, and cognitive vulnerability $(\mathrm{p}<0.05)$ (Table 1 and Graph 1$)$. We also observed significant association between dental decay and level of dental fear (Table 2). Our results were in correlation with the results obtained by Carrillo-Diaz et $\mathrm{al}^{8}$ who reported similar findings in their study. Pohjola et $\mathrm{al}^{14}$ assessed whether various dental parameters were associated with dental anxiety and fear. They analyzed 8,028 adults belonging to age group of 30 years and above. A total of 6,335 home interviews were conducted and clinical oral examination was done. Dental fear was assessed by the authors by conducting this questionnaire-based survey. An association of dental fear was observed with all the dental parameters with exception of number of restored teeth. They also observed positive association between dental fear and quantity of decayed teeth, which were observed to be independent of age and gender of the individual. Age adjusted the relationship between dental dread and the number of absent and sound teeth. Among the most seasoned age gathering, the quantities of lost and sound teeth were decidedly connected with dental dread while being contrarily related among the youngest age gathering. They observed that in comparison with the individuals with lower fear, individuals with high dental fear exhibited poorer dental condition. Neither gender nor dental participation influences the relationship between dental dread and dental condition. According to the birth year, variation was noticed while assessing the correlation between dental fear and quantity of missing and sound teeth. Carrillo-Diaz et $\mathrm{al}^{8}$ evaluated 250 pediatric patients and assessed various psychosocial parameters in children attending dental clinics. They particularly focused on the dental-related cognitive vulnerability perception and beliefs related to prevention of dental problems. A questionnaire was framed which was completely filled by 250 Spanish schoolchildren. Experienced pedodontists were hired by them for evaluation of the oral health status. Children were found to be reluctant to visit dental clinics due to vulnerability-related cognitions and inappropriate dental prevention beliefs. In addition, a higher frequency of tooth decay was found to be correlated with the interaction between the cognitive vulnerability perceptions and dental prevention beliefs. The sporadic example of dental visit, related with frightful and negative dental

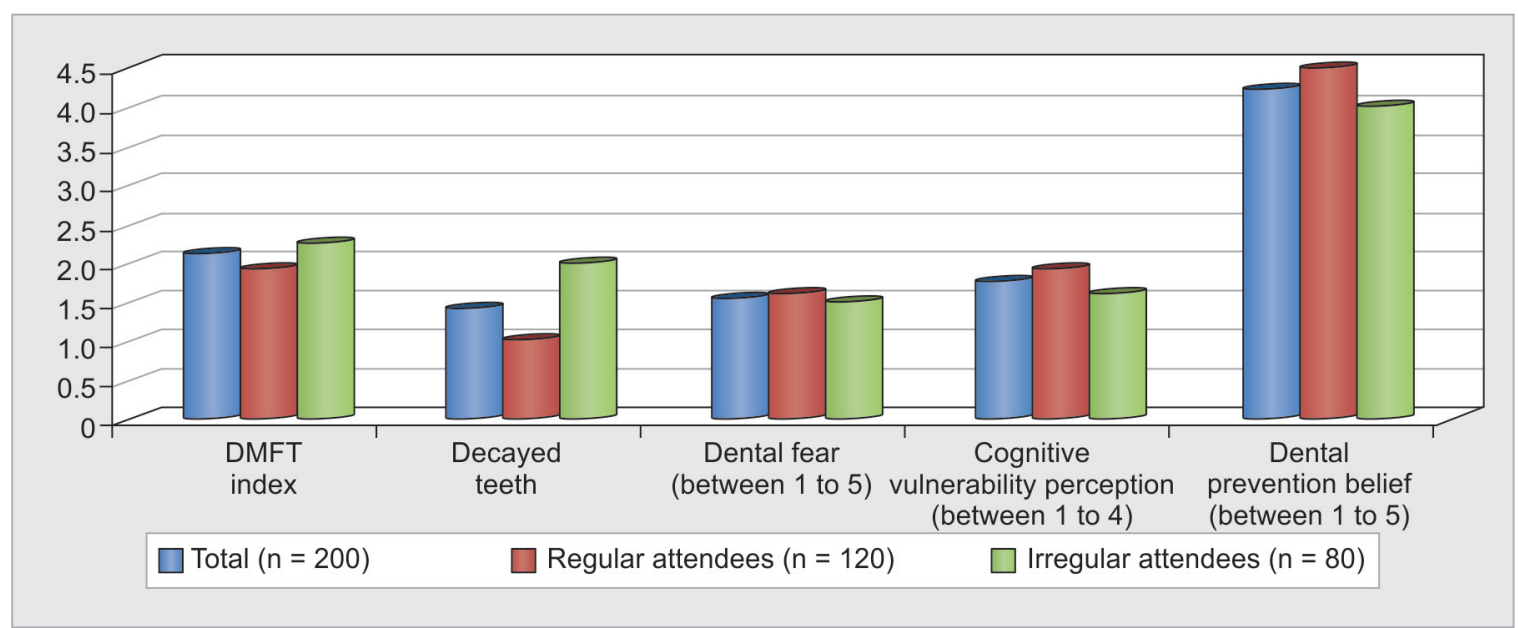

Graph 1: Mean of various parameters between regular and irregular attendees 
counteractive action perceptions, represented $20 \%$ of the impacts of these factors on dental caries. The blend of more prominent intellectual observations and low familiarity with the advantages of dental counteractive action expanded the danger of dental caries. Pediatric patients with this type of profile additionally had a tendency to exhibit a more lacking example of dental participation. From the results, the authors concluded that preventive oral well-being projects would profit by considering the part of kids' insights on their oral well-being propensities and dental well-being.

Prasai Dixit et $\mathrm{al}^{15}$ assessed the incidence of dental caries and various caries preventive practices among schoolchildren. The authors conducted a cross-sectional epidemiological study that involved five government primary schools of Chitwan District. They prepared a questionnaire and gave it to a total of 131 participants aged from 8 to 16 years. Among 361 schoolchildren, clinical and oral examination was done following criteria set by the WHO in 1997 for the diagnosis of caries. Caries predominance for 5- to 6-year-old children was over the objectives prescribed by the WHO and the Federation of Dentistry Global of fewer than half caries-free youngsters. Among individuals of 5 to 6 years of age and individuals of 12 to 13 years of age, caries prevalence was found to be 52 and $41 \%$ respectively. Among individuals of 5 to 6 years of age and 12 to 13 years of age, the mean $\mathrm{dmft} / \mathrm{DMFT}$ was found to be $0.59,0.31$ and $0.52,0.84$ respectively. Around $31 \%$ of 8 to 16 -year-old school kids who have taken an interest in the study revealed having experienced oral torment. In all the individuals, it was found that there is need for treating the decayed teeth. Around $76 \%$ kids saw teeth as an imperative part of general well-being and $75 \%$ detailed it was required to eat. More than $90 \%$ of the involved individuals were found to have never visited a dentist. For cleaning of the teeth, toothpaste was used by approximately $85 \%$ of the individuals. Albeit $61 \%$ youngsters answered to have gotten oral well-being instruction, $82 \%$ kids did not think about fluoride and its advantage on dental well-being. From the results, the authors concluded that there is a need for cheaper school-based intervention for prevention of dental caries.

Patients' previous experiences in dental clinic and their psychosocial milieu impose major hurdle to access dental care. These factors unite jointly to create barriers decreasing the patients' aptitude to avail dental and oral health care. ${ }^{16}$

\section{CONCLUSION}

In light of the above results, the authors conclude that various psychosocial parameters, by affecting the dental visits, are associated with outcome of oral health measures. However, future studies are recommended in this field of dentistry for further exploration of results.

\section{REFERENCES}

1. Locker D. Psychosocial consequences of dental fear and anxiety Community Dent Oral Epidemiol 2003 Apr;31(2):144-151.

2. Eitner S, Wichmann M, Paulsen A, Holst S. Dental anxiety - an epidemiological study on its clinical correlation and effects on oral health J Oral Rehabil 2006 Aug;33(8):588-593.

3. Samorodnitzky GR, Levin L. Self-assessed dental status, oral behavior, DMF, and dental anxiety J Dent Educ 2005 Dec;69(12):1385-1389.

4. Wisløff TF, Vassend O, Asmyhr O. Dental anxiety, utilisation of dental services, and DMFS status in Norwegian military recruits Community Dent Health 1995 Jun;12(2):100-103.

5. Sohn W, Ismail AI. Regular dental visits and dental anxiety in an adult dentate population J Am Dent Assoc 2005 Jan;136(1):58-66.

6. Thomson WM, Stewart JF, Carter KD, Spencer AJ. Dental anxiety among Australians Int Dent J 1996 Aug;46(4): 320-324.

7. Armfield JM, Stewart JF, Spencer AJ. The vicious cycle of dental fear: exploring the interplay between oral health, service utilization and dental fear BMC Oral Health 2007 Jan;7:1.

8. Carrillo-Diaz M, Crego A, Armfield JM, Romero M. Dental fear-related cognitive vulnerability perceptions, dental prevention beliefs, dental visiting, and caries: a cross-sectional study in Madrid (Spain) Community Dent Oral Epidemiol 2015 Aug;43(4):375-384.

9. Chhabra N, Chhabra A, Walia G. Prevalence of dental anxiety and fear among five to ten year old children: a behaviour based cross sectional study Minerva Stomatol 2012 Mar;61(3): 83-89.

10. Lee CY, Chang YY, Huang ST. Prevalence of dental anxiety among 5- to 8-year-old Taiwanese children J Public Health Dent 2007 Winter;67(1):36-41.

11. Klingberg G, Broberg AG. Dental fear/anxiety and dental behaviour management problems in children and adolescents: a review of prevalence and concomitant psychological factors Int J Paediatr Dent 2007 Nov;17(6):391-406.

12. Hakeberg M, Berggren U, Gröndahl HG. A radiographic study of dental health in adult patients with dental anxiety Community Dent Oral Epidemiol 1993 Feb;21(1):27-30.

13. Armfield JM, Slade GD, Spencer AJ. Dental fear and adult oral health in Australia Community Dent Oral Epidemiol 2009 Jun;37(3):220-230.

14. Pohjola V, Lahti S, Vehkalahti MM, Tolvanen M, Hausen H. Age-specific associations between dental fear and dental condition among adults in Finland Acta Odontol Scand 2008 Oct;66(5):278-285.

15. Prasai Dixit L, Shakya A, Shrestha M, Shrestha A. Dental caries prevalence, oral health knowledge and practice among indigenous Chepang school children of Nepal BMC Oral Health 2013 May;13:20.

16. Freeman R. The psychology of dental patient care: barriers to accessing dental care: patient factor Br Dent J 1999 Aug;187:141-144. 